\title{
Supporting Information: Dimensionality-Reduced Fermi Level Pinning in Coplanar 2D Heterojunctions
}

\author{
Henry Yu ${ }^{1,2}$, Sunny Gupta ${ }^{2}$, Alex Kutana ${ }^{2}$, Boris I. Yakobson ${ }^{1,2,3, \dagger}$ \\ ${ }^{1}$ Applied Physics Program, Rice University, Houston, TX, 77005 \\ ${ }^{2}$ Department of Materials Science and Nanoengineering, Rice University, Houston, TX, 77005 \\ ${ }^{3}$ Department of Chemistry, Rice University, Houston, TX, 77005 \\ †email: biy@rice.edu
}

\section{Equivalent circuit model for 3D $\mathrm{M} \mid \mathrm{S}$ junction}

The equivalent circuit model is an idealization dividing the junction into three distinct parts - metal, semiconductor, and interface. In a real physical junction, each part is defined by its electronic states, e.g. the interface is defined by its local states. The potentials on the circuit nodes correspond to average potential in the bulk of the metal or semiconductor, as well as the potential at the interface determining its level occupancies. The neutrality point of the interface is the position of the Fermi level at which interface states do not take part in the formation of a local dipole, i.e. have zero charge. The nature of interface states could be dangling bonds or metal-induced gap states (MIGS). In the latter case, the nature of MIGS is determined by bulk semiconductor properties, the MIGS neutrality point is universal (i.e. independent on the kind of metal or semiconductor surface orientation), and corresponds to energy at which MIGS character changes from valence- to conduction band-like. ${ }^{6}$
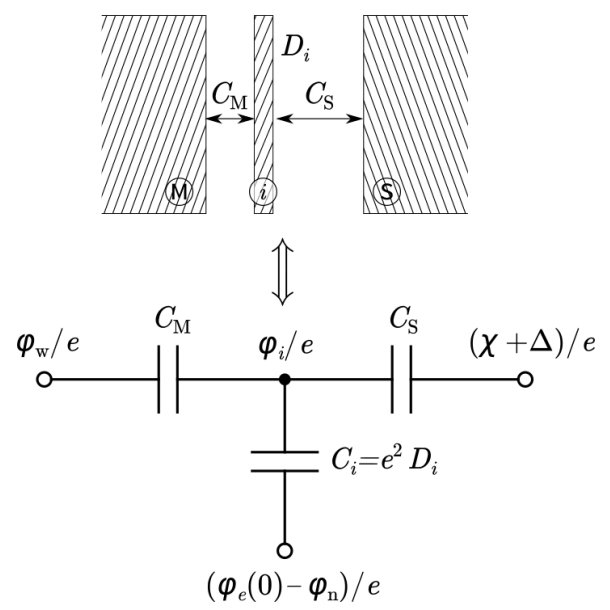

FIG. S1 Description of the metal-semiconductor junction using an equivalent circuit diagram (with unit charge $e$ restored). Metal, semiconductor and interface parts are shown at the top. $C_{\mathrm{M}}$ and $C_{\mathrm{S}}$ are the metal-interface and semiconductor-interface geometric capacitances, respectively, and $C_{i}$ is quantum capacitance defined as $C_{i}=e^{2} D_{i}$, where $D_{i}$ is the (constant) density of interface states. Voltages on the equivalent circuit diagram at the bottom are defined in the main text.

After making the contact, charge redistribution occurs, leading to the buildup of electrostatic potential. Charges on metal, semiconductor, and interface are found by minimizing the total energy, subject to neutrality constraint. ${ }^{7}$ The charges are then the same as those in the equivalent circuit fig. S1, with battery voltages given by the differences of the intrinsic workfunctions (WF). The intrinsic WF (battery potentials) of the metal, semiconductor and interface are termed $\varphi_{\mathrm{w}}, \chi$, and $\varphi_{e}(0)-\varphi_{\mathrm{n}}$, respectively. The geometric classical capacitance of each part is determined from its spatial charge distribution by the usual definition as a proportionality constant between charge and potential. The band energy is $E_{\text {band }}=\sigma^{2} / 2 D e^{2}+(\sigma / e) \varepsilon_{\text {neut }}$, and the electrostatic energy is $E_{\mathrm{ES}}=\sigma^{2} / 2 C$, where $e=|e|$ is elementary charge. Here $D$ and $\varepsilon_{\text {neut }}$ are the (constant) density of states and neutrality level of the material, respectively, and $C$ is geometric capacitance. The total energy of the junction is the sum of energies of its three parts. Assuming very large DOS for metal and semiconductor, the following charges are obtained after total energy minimization: $e \sigma_{M}=\frac{C_{M} C_{i}}{C_{\Sigma}}\left(\varphi_{e}(0)-\varphi_{n}-\varphi_{w}\right)-\frac{C_{M} C_{S}}{C_{\Sigma}}\left(\varphi_{w}-(\chi+\Delta)\right)$ 
$e \sigma_{S}=\frac{C_{s} C_{i}}{C_{\Sigma}}\left(\varphi_{e}(0)-\varphi-(\chi+\Delta)\right)+\frac{C_{M} C_{s}}{C_{\Sigma}}\left(\varphi_{w}-(\chi+\Delta)\right)$

$e \sigma_{i}=\frac{C_{M} C_{i}}{C_{\Sigma}}\left(\varphi_{w}-\varphi_{e}(0)+\varphi_{n}\right)+\frac{C_{s} C_{i}}{C_{\Sigma}}\left(\chi+\Delta-\varphi_{e}(0)+\varphi_{n}\right)$

Here, $C_{\mathrm{M}}$ and $C_{\mathrm{S}}$ are the metal-interface and semiconductor-interface geometric capacitances, respectively, and $C_{i}$ is quantum capacitance defined as $C_{i}=e^{2} D_{i}$, and $C_{\Sigma}=C_{\mathrm{M}}+C_{\mathrm{S}}+C_{i}$. These charges are described by the equivalent circuit fig. S1, equating battery potentials with intrinsic WF differences. At equilibrium the interface will be charged, with an adjusted WF $\varphi_{i}=\varphi_{e}(0)-\varphi_{\mathrm{n}}+e \sigma_{i} / C_{i}$.

\section{Further details of multi-scale model}

\section{1 atomic structure}

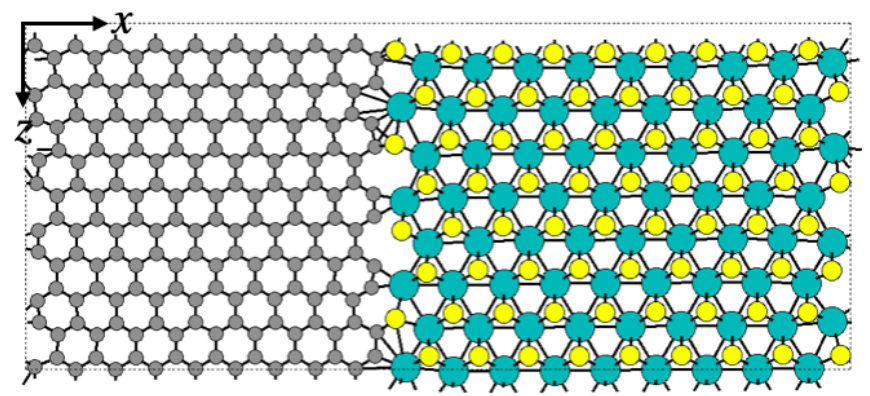

FIG. S2 top view of atomic structure of the $\mathrm{Gr} \mid \mathrm{MoS}_{2}$ interface used in the multi-scale model.

\section{2 flow chart of continuum model}

The multi-scale modeling code is available upon request. For reference we also give a detailed flowchart for the numerical routine solving eq. 2-3 in the main text, as shown below and illustrated in fig. S3.

1. Start

2. Begin with trial solution to potential $\varphi_{e}(x, y)$

3 . Find the electron $(n(x))$ and hole $(p(x))$ densities from $\varphi_{e}(x, y)$ according to rigid band approximation (eq. $3)$. The quasi-Fermi levels for electron $\left(E_{\mathrm{F} n}\right)$ and hole $\left(E_{\mathrm{F} p}\right)$ should be adjusted such that $E_{\mathrm{F} n}-E_{\mathrm{F} p}=V$, and also the total charge should be neutral.

4. Find the new potential $\varphi_{e}(x, y)$ according to Poisson equation as in eq. 2 .

5. Check if error $\int\left|\varphi_{e, \text { new }}(x)-\varphi_{e, \text { old }}(x)\right| d x<\delta$ is below tolerance. If not, go to 6.; if so, go to 7.

6. Create new solution from mixing $\varphi_{e}=(1-\alpha) \varphi_{e, \text { new }}+\alpha \varphi_{e, \text { old. }}$. Go back to 3 .

7. End

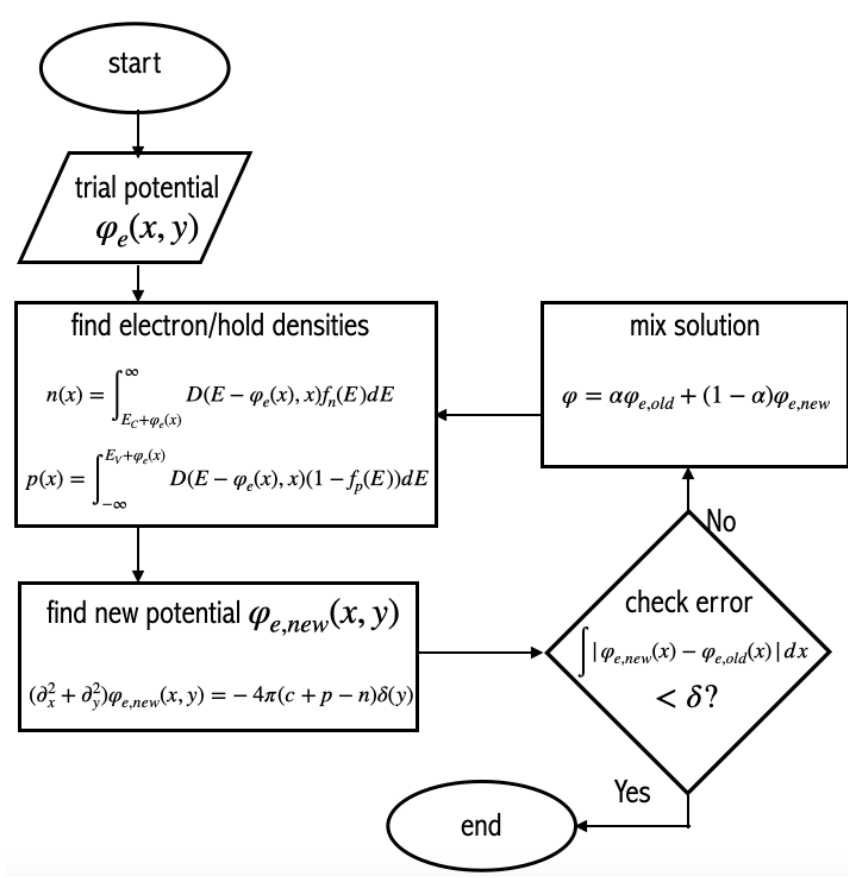

FIG. S3 Flow chart for solving the multi-scale model described in eq. 3 in main text.

Note that for the most accurate representation one should solve both the electrostatics and transport self-consistently. However, to make our treatment computationally efficient, and allows us to obtain accurate potential and charge profiles, we follow Shockley's analysis of $p-n$ junctions (ref. 35 main), wherein the full charge/potential profile under finite bias $V$ can be very efficiently calculated under a "pseudo-equilibrium" treatment. In this treatment, the current is assumed zero $(j=0)$ and the quasi-Fermi level for electrons and holes are held at $E_{\mathrm{F} n}$ and $E_{\mathrm{F} p}$ respectively, with a difference $E_{\mathrm{F} n}-E_{\mathrm{F} p}=V$ between them. Carrier transport (finite $j$ ) will cause a negligible change to the charge/potential profile with respect to this pseudo-equilibrium result.

\subsection{Enlarged potential profiles}




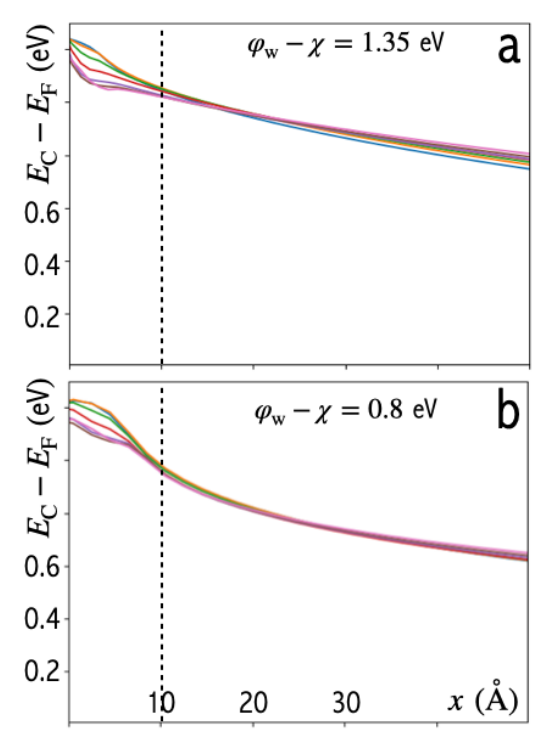

FIG. S4 Enlarged potential profile plots from fig. 3 in main text, for (a) $\varphi_{\mathrm{w}}-\chi=1.35 \mathrm{eV}$ and for (b) $\varphi_{\mathrm{w}}-\chi=0.8 \mathrm{eV}$. Dashed line marks the extent of the interface states (from the interface position at $x=0$ ).

Finally, we include here the enlarged potential profiles calculated from the multi-scale model (fig. 3 main). The dashed line marks the location where the interface DOS ends.

\section{Macroscopic averaging}

\subsection{Conservation of dipole moment}

Within the MA method, a normalized kernel $w(r)$ is used to convolve with the microscopic fields $f(r)$ as $F(r)=\int d r^{\prime} f(r) w\left(r-r^{\prime}\right)$ to suppress the oscillating components and obtain the macroscopic field $F(r)$; here $f(r)$ can be the charge $\rho(r)$ or potential energy $\varphi(r)$, with periodicity of the crystal lattice. In Fourier space this procedure is equivalent to applying a low-pass filter $\tilde{w}(k)$ to the Fourier transformed signal $\tilde{f}(k)$. The periodic features of $f(r)$ produce singularities in the Fourier space $\tilde{f}(k)$, therefore, the kernel $\tilde{w}(k)$ should be chosen such that the singular terms at the reciprocal lattice points $k=G$ are suppressed. ${ }^{1}$ Most importantly, the dipole moment of an interface charge dipole is conserved under this procedure; this can be proved as the following. For an arbitrary kernel $w(r)$, the dipole moment of the MA charge $R(r)$ can be calculated as $P=\int d r R(r) r=\int d r d k r \tilde{R}(k) e^{i k \cdot r}=-2 \pi i \tilde{R}^{\prime}(0)$. From convolution theorem we know that $\left.\tilde{R}^{\prime}(k)=\tilde{(\rho}(k) \widetilde{w}(k)\right)^{\prime}$; the kernel function $w(r)$ is normalized therefore $\tilde{w}(0)=1$, and $\tilde{n}(0)=0$ for zero total charge. Combining these conditions we have the dipole moment $P=-2 \pi i \tilde{R}^{\prime}(0)=-2 \pi \tilde{i \rho^{\prime}}(0)$, invariant of the choice of $w(r)$. Normally, as long as the mollifier $w(r)$ is wide and smooth compared to atomic distances, the end results do not depend on the choice of $w(r) .{ }^{1}$ However, in practical ab initio calculations of material interfaces, a macroscopically large system is unaffordable. Therefore, we cannot use a wide enough kernel $w(r)$ and the end results will depend on the choice of kernel and should be interpreted with care. Following ref. ${ }^{2}$ we choose $w(r)$ as a uniform window with length of the unit cell period, which can precisely suppress the atomic features. As mentioned above, the choice of this window is not unique, but the dipole moment and the band alignment is invariant to the choice of $w(r)$.

\subsection{Successive averaging}

In applying MA to a heterojunction, a window function $w(x)$ should be chosen such that atomistic details of both materials can be suppressed. As proposed in the main text, this can be done by successive MA with $s_{G}$ and $s_{M}$, square waves with period equal to lattice size of graphene $\left(s_{G}\right)$ and $\mathrm{MoS}_{2}\left(s_{M}\right)$. More explicitly,

$$
\begin{gathered}
F(x)=\iint f(u) s_{G}(v-u) s_{M}(x-v) d u d v \\
=\int d u f(u) \int s_{G}(v-u) s_{M}((x-u)-(v-u)) d v \\
=\int d u f(u) w(x-u)
\end{gathered}
$$

where $w(x)=\int s_{G}(v) s_{M}(x-v) d v$, the convolution of both window functions. Figure S5 plots the $s_{G}(x)$ (fig. S5a), $s_{M}(x)$ (fig. S5b), and $\left(s_{G}{ }^{*} s_{M}\right)(x)$ (fig. S5c) for reference.

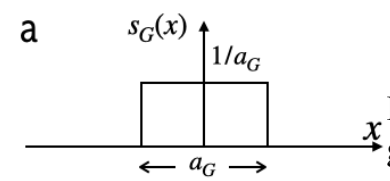

FIG. S5 (a) square wave for graphene lattice (b) square wave for $\mathrm{MoS}_{2}$ lattice (c) convolution b $s_{M}(x) \uparrow \quad$ of $s_{G}$ and $s_{M}$, resulting in a

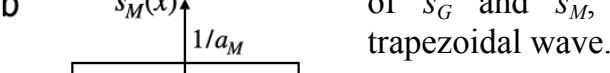
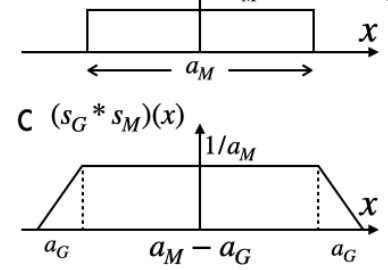


\section{Interface dipole in $\mathrm{WSe}_{2} \mid \mathrm{MoSe}_{2}$ interface}

We present below an example of applying the MA method on the $\mathrm{WSe}_{2} \mid \mathrm{MoSe}_{2}$ interface to calculate the band offset. The band offset, for which for definiteness we take the difference in positions of the valence band edges $E_{\mathrm{V}}$, is split into two contributions: the potential lineup of the MA potential $\Delta V$ from the interface calculation, and the lineup of valence band edges $\Delta E_{\mathrm{V}}$ referenced to the average potential of individual materials from primitive cell calculations. ${ }^{2-4} \mathrm{~A}$ new feature in calculations of 2D junctions is that, when applying the MA method to calculate band alignment, the reference vacuum level $V_{\text {vac }}$ should be taken into account. Moreover, in typical plane wave basis models periodic boundary conditions (PBC) in the $y$-direction are enforced, which results in spurious Coulomb interactions among the periodic layers in the $y$-direction and an ill-behaving vacuum level. As shown previously, ${ }^{5}$ this can lead to incorrect band alignment and has very slow convergence, requiring a vacuum length up to $L_{y} \sim 100 \AA$ for correct band alignment. This slow convergence can be explained through simple electrostatic considerations. The error in band alignment is a result of the erroneous potential step $\Delta V_{\text {err }}$ created by the periodic images of the dipoles in $y$ direction. Given an interfacial dipole of moment per width $\tau_{2 \mathrm{D}}$, a vacuum length $L_{y}$ gives an areal dipole density of $\tau_{2 \mathrm{D}} / L_{y}$, which creates a potential step $\Delta V_{\text {err }}=4 \pi \tau_{2 \mathrm{D}} / L_{y}$. Hence, the error decays slowly as $\sim \tau_{2 \mathrm{D}} / L_{y}$. By taking the ill-behaving vacuum potential into account, the $2 \mathrm{D}$ limit $L_{y} \rightarrow \infty$ result can be accurately obtained for a moderate vacuum size (e.g. $L_{y}=15 \AA$ in our case).
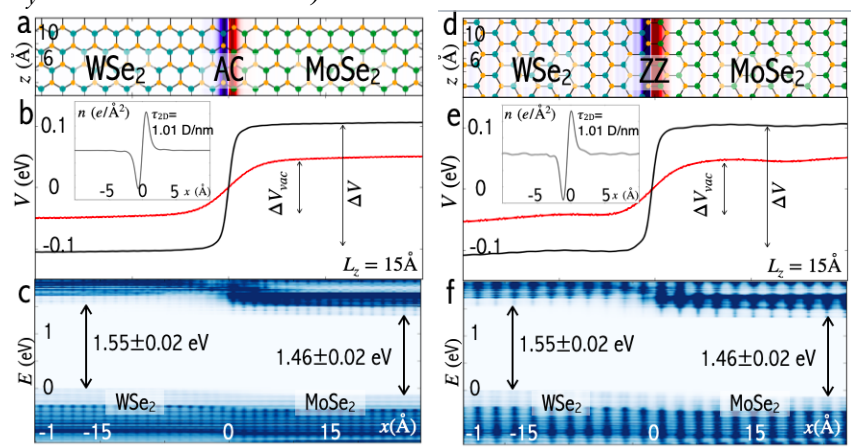

FIG. S6 Calculated atomic structures, interface dipoles, average electrostatic potentials, and LDOS of armchair (a)-(c) and zigzag (d)-(f) 2D lateral heterojunctions of $\mathrm{WSe}_{2} \mid \mathrm{MoSe}_{2}$. (a)(d) atomic structures of the armchair and zigzag interfaces, respectively, with the charge dipole (red-blue) shown in the background. (b)(e) the electrostatic potential at vacuum $V_{\text {vac }}(x)$ (red line), and MA electrostatic potential $V(x)$ (black line, see text). (c)(f) LDOS along $x$ direction displaying the electronic structures of both materials and interface.

Figure S6a shows the atomic structure of the AC interface. In order to obtain the interface dipole charge $\sigma_{2 \mathrm{D}}(x)$, we average the $a b$ initio electronic charge $n(x, y, z)$ along the periodic $z$ direction, apply MA along $x$, and integrate over $y$ to arrive at a $2 \mathrm{D}$ distribution shown in the background of fig. S6a. The MA interface charge profile is shown in the inset of fig. S6b, clearly displaying a dipolar shape with moment $\tau_{2 \mathrm{D}}=0.021 e$ or 0.101 Debye/ $\AA$ per width of interface. Note that Bader charge of interface atoms gives a vanishingly small charge transfer of $1.5 \mathrm{me} / \AA$. The MA potential profiles are shown in fig. S6b. The black solid curve shows $V(x)$, the MA electrostatic potential from the supercell calculation, obtained from the Hartree potential $\varphi_{\mathrm{H}}(x, y, z)$ by averaging over $y$ and $z$ directions and applying MA along $x$, with a step of $\Delta V=0.21 \mathrm{eV}$. The red solid curve shows the vacuum potential $V_{\mathrm{vac}}(x)$, obtained from $\varphi_{\mathrm{H}}(x, y, z)$ by averaging over $z$, taking $y=L_{y} / 2$. In order to be used as a reference, the vacuum potential should approach a constant away from the 2D materials. Here the vacuum potential clearly shows a step, which is a result of the spurious Coulomb interactions from the periodic interface dipoles in the $y$ direction. In the 2D limit $L_{y} \rightarrow \infty$ this vacuum potential converges to a constant rather slowly $\left(\Delta V_{\mathrm{vac}} \sim \tau_{2 \mathrm{D}} / L_{z}\right)$; for $L_{y}=15 \AA$, there is a step of $\Delta V_{\mathrm{vac}}=0.09 \mathrm{eV}$. Accounting for the unconverged vacuum potential, the potential lineup can be calculated as $\Delta V^{*}=\Delta V-\Delta V_{\mathrm{vac}}=0.12 \mathrm{eV}$. When referenced to their own average potential, the valence band edges of each material give a difference $\Delta E_{\mathrm{V}}=-0.31 \mathrm{eV}$. Combining the two terms, the total valence band offset of $-0.19 \mathrm{eV}$ is obtained for a $\mathrm{WSe}_{2} \mid \mathrm{MoSe}_{2} \mathrm{AC}$ interface. Same values can also be obtained independently from band positions of individual materials. As mentioned above, there is a natural common potential reference for $2 \mathrm{D}$ materials; thus their band alignments are well defined even before the interface is made. Referencing the valence bands of pure $\mathrm{WSe}_{2}$ and $\mathrm{MoSe}_{2}$ to their vacuum levels give $E_{\mathrm{V}}{ }^{\mathrm{WSe} 2}=-5.16 \mathrm{eV}$ and $E_{\mathrm{V}}{ }^{\mathrm{MoSe} 2}=-5.35 \mathrm{eV}$, which gives a band offset of $-0.19 \mathrm{eV}$, in perfect agreement with results from the MA technique. This confirms our analysis above, that band alignments in 2D junctions are well defined and unaffected by the $2 \mathrm{D}$ interface dipole.

Apart from the lineup of potentials and interface charge profile, we also show the local density of states (LDOS) as shown in fig. S6c, calculated as 
$d(E, x)=\left\langle\int d y \sum_{n k} \delta\left(E-E_{n k}\right)\left|u_{n k}(\vec{r})\right|^{2}\right\rangle{ }_{z}$ where $u_{n k}$ is the Bloch wave function at given k-point $k$ and band number $n$, and the sum is taken over the Brillouin zone and all bands. For the delta function we approximate it as $\delta(E) \approx u(E)$, a narrow uniform window with a width of $0.02 \mathrm{eV}$. By measuring the band edges in the LDOS we found that the bulk PBE band gap values of $\mathrm{WSe}_{2}$ $(1.54 \mathrm{eV})$ and $\mathrm{MoSe}_{2}(1.44 \mathrm{eV})$ are restored at the far left and right side. In addition, there is a smooth transition in the band edges across the interface of width $\sim 10 \AA$, showing that there is considerable orbital hybridization between the two materials within this region. This is consistent with the presence of an interface dipole since there must be some orbital hybridization for any charge transfer to occur between two semiconductors. Similar results for band alignment are obtained for the $\mathrm{ZZ}$ interface, as shown in fig. S6d-f.

\section{Pinning strength in $2 \mathrm{D} \mathrm{M} \mid \mathrm{S}$ contacts}

Due to the wide depletion region, the current in eq. 4 for the $2 \mathrm{D} \mathrm{M} \mid \mathrm{S}$ contact can be approximated as

$j \approx g_{0} k_{B} T e^{\left(V-\varphi_{B 0}\right) / k_{B} T} X$; here $\varphi_{\mathrm{B} 0}$ is the original SBH and $X=\int_{0}^{\varphi} e^{-\frac{2}{\hbar} \sqrt{2 m(\varphi-E)} l} e^{-E / k_{B} T} \frac{d E}{k_{B} T}+e^{-\varphi / k_{B} T}$ is an additional tunneling factor due to the dipole potential of energy-height $\varphi=\varphi_{\mathrm{B}}-\varphi_{\mathrm{B} 0}$ and space-width $l_{p}$; the effective mass in $\mathrm{MoS}_{2}$ is $m=0.48 m_{0}$ (ref. 35 main) ( $m_{0}$ the electron mass). Due to the $\sim 1 / x$ trend of a $2 \mathrm{D}$ dipole potential, we can estimate $l_{p} \sim l \varphi / \varphi_{0}$ where $\varphi_{0}=0.1$ $\mathrm{eV}$ is a potential cut off. The $\mathrm{SBH}$ is therefore $\varphi_{\mathrm{B}}=k_{\mathrm{B}} T \ln \left(g_{0} k_{\mathrm{B}} T / j_{0}\right)=\varphi_{\mathrm{B} 0}-k_{\mathrm{B}} T \ln X$. With these estimates and re-expressing all energies in thermal units $\epsilon=E / k_{\mathrm{B}} T$, $\varphi^{*}=\varphi / k_{\mathrm{B}} T$ one can find that $X=X\left(\varphi^{*}, l / \lambda_{\mathrm{D}}\right)$, that the tunneling factor $X$ depends on $\phi$ and the parameter $l / \lambda_{\mathrm{D}}$, with $\lambda_{\mathrm{D}}=\left(2 \pi \hbar^{2} / m k_{\mathrm{B}} T\right)^{1 / 2} \sim 63 \AA$ as the thermal de Broglie wavelength (ref. 27 in main). Essentially, the ratio $l / \lambda_{\mathrm{D}}$ determines the behavior of the thermal carriers in the presence of the potential barrier. For a wide barrier $l / \lambda_{\mathrm{D}} \gg 1$ the carriers behave classically and are blocked by the barrier; hence, the second term in $X$ dominates, i.e. $X \approx e^{-\varphi / k_{B} T}$ and therefore is fully pinned $\left(\varphi_{\mathrm{B}} \approx E_{\mathrm{C}}-\varphi_{\mathrm{n}}\right)$. For the $\mathrm{Gr} \mid \mathrm{MoS}_{2}$ contact in our example with $l \sim 10 \AA$ gives a moderate value $l / \lambda_{\mathrm{D}}=0.16$ and hence partial pinning. Finally, for a thin interface $\left(l / \lambda_{\mathrm{D}} \ll 1\right)$, the carriers tunnel through the thin quantum barrier, resulting in zero pinning.

\section{References}

(1) Ashcroft, N. W.; Mermin, N. D. Solid State Physics; Holt, Rinehart and Winston, NY, 1976.

(2) Baldereschi, A.; Baroni, S.; Resta, R. Band Offsets in Lattice-Matched Heterojunctions: A Model and First-Principles Calculations for GaAs/AlAs. Phys. Rev. Lett. 1988, 61, 734-737. https://doi.org/10.1103/PhysRevLett.61.734.

(3) Peressi, M.; Baroni, S.; Resta, R.; Baldereschi, A. Tuning Band Offsets at Semiconductor Interfaces by Intralayer Deposition. Phys. Rev. B 1991, 43, 7347-7350. https://doi.org/10.1103/PhysRevB.43.7347.

(4) Ruini, A.; Resta, R.; Baroni, S. Effects of Interface Morphology on Schottky-Barrier Heights: A Case Study on Al/GaAs(001). Phys. Rev. B 1997, 56, 14921-14924. https://doi.org/10.1103/PhysRevB.56.14921.

(5) Zhang, J.; Xie, W.; Zhao, J.; Zhang, S. Band Alignment of Two-Dimensional Lateral Heterostructures. 2D Mater. 2017, 4, 015038.

(6) Tersoff, J. Schottky Barrier Heights and the Continuum of Gap States. Phys. Rev. Lett. 1984, 52, 465-468. https://doi.org/10.1103/PhysRevLett.52.465.

(7) Luryi, S. Quantum Capacitance Devices. Appl. Phys. Lett. 1988, 52, 501-503. http://dx.doi.org/10.1063/1.99649. 